\title{
Membrane Computing as the Paradigm for Modeling Systems Biology
}

\author{
Ravie Chandren Muniyandi and Abdullah Mohd. Zin \\ Research Center for Software Technology and Management, \\ Faculty of Information Science and Technology, \\ University Kebangsaan Malaysia, 43600 Bangi, Selangor, Malaysia
}

Received 2012-09-24, Revised 2013-02-15; Accepted 2013-03-11

\begin{abstract}
Membrane computing is a field in computer science that is inspired from the structure and the processes of living cells and is being considered as an alternative in solving the limitations in conventional mathematical approaches by taking into consideration its essential features that are of interest for research in systems biology. Advancements in computability make it feasible to handle huge volumes of data in biology and propose a new and better approach using a discreet computer science model, such as membrane computing. In this respect, membrane-computing abilities, to enhance the understanding of the system level of biological systems, have been explored. This study discusses experiences in applying membrane computing in modeling biological systems as well as possibilities of incorporating membrane computing into other computer science paradigms to enhance the use of membrane computing in systems biology. Experiences in modeling aspects of systems biology with membrane computing demonstrate additional advantages and possibilities compared with conventional methods. However, they are not yet used widely to model or simulate biological processes or systems. A general framework of modeling and verifying biological systems using membrane computing is essential as a guideline for biologists in their research in systems biology.
\end{abstract}

Keywords: Membrane Computing, Prey Predator Population, Discrete Systems

\section{INTRODUCTION}

Membrane computing or P Systems (Paun et al., 2010) is a branch of computer science that conceptualizes ideas and models of computation based on the structure and the processes of living cells. It draws inspiration from the manner cells are organized in complex biological structures. Membrane computing is also denoted to computing models with distributed and parallel processing, in which multisets of symbol-objects in different compartments are delimited by membranes by applying evolution rules.

This description applies to a very basic type of membrane system. Different variants of membrane computing have also been studied, but the fundamental element of membrane computing is its membrane structure (Paun et al., 2010). Membranes can be arranged in a hierarchical structure as in a cell, or in an internetwork of different membranes as in a tissue. In biology, a membrane is conceptualized as a threedimensional vesicle, but the notion is generalized to infer a membrane as a separator of two regions or compartments. The finite inside is delimited by the membrane and the infinite outside is linked to the environment. This concept provides the opportunity for a selective communication between the two regions in the biological systems. Investigations on membrane computing did not just provide different models, but also portrayed membrane computing as formalism in devising compartmentalized models.

Most mathematical models of biological systems were created using continuous mathematics, especially a system of Ordinary Differential Equations (ODE) (Blanchard et al., 2011), in which variations in the $\begin{aligned} \text { Corresponding Author: } & \text { Ravie Chandren Muniyandi, Research Center for Software Technology and Management, } \\ & \text { Faculty of Information Science and Technology, University Kebangsaan Malaysia, } 43600 \text { Bangi, } \\ & \text { Selangor, Malaysia }\end{aligned}$ 
concentration of each chemical substance or object are modeled as global entities. This approach makes it difficult to develop modular and scalable designs. As a consequence, researchers in computational biology have encountered limitations in modeling the behaviors and the characteristics of biological systems with respect to models that are based on analytic, continuous mathematics.

An important advancement in computability, which makes it feasible to handle huge volumes of data and which is already available in biology, states that a new period of mathematization of biology is possible by means of a discreet computer science model, such as membrane computing (Paun et al., 2010). Membrane computing is considered as an alternative in solving the limitations in ODE by taking into consideration its essential features that could be used in biological applications. Some of those features (Paun et al., 2010) are the following: (a) Distribution capability-the composition of local behaviors enables nonlinear systempart interaction and emergent behavior; (b) Discreet nature- biological processes are discreet and are offered as alternative to the system of differential equation, which cannot cover more than the local processes and the imprecise character of the processes; (c) Algorithmic representation- membrane computing is a computability model that is the same as Turing machine representations of algorithms and is, thus, easily simulated on computers; (d) Scalability/extensibility-modularity that is available in membrane computing provides this facility and to counter the difficulties in differential equation; (e) Transparency- multiset rewriting rules in membrane computing are reaction equations without any complex notation or behavior, as used in biochemistry; (f) Massive parallelism- a general behavior of a biological system; (g) Non-determinism- a set of rules localized in certain compartments, without any imposed order in the implementation of rules and (h) Communication- the interaction between compartments.

Recently, there has been a growing interest in the use of membrane computing, a theoretical paradigm abstracting from the structure and the functionality of a living cell, as a model and simulator of cellular phenomena, thereby producing formal specifications defined in a language that is close to that of molecular biology, whose information is easy for biologists to interpret. In this respect, there have been investigations into modeling various aspects of molecular and cellular biology.

\section{MATERIALS AND METHODS}

\subsection{Modeling with Membrane Computing}

Applying membrane computing to modeling biological systems has been taken from the experiences of authors in modeling biological systems, as well as the experiences in other research.

\subsection{Integrating Membrane Computing with Computer Science Paradigm}

Attempts to integrate membrane computing elements with computer science paradigms are analyzed based on the attempts made by several researchers in the area of computer science.

\section{RESULTS}

\subsection{Experiences in Modeling Biological Systems with Membrane Computing}

Systems biology (Meyers, 2012) is a growing field in research that develops the understanding of the system level of biological systems. The fundamental nature of a system is based on its dynamics and cannot be explained by simply specifying components of the system. However, merely considering the system structure, such as network topologies, is inappropriate without sufficiently addressing the varieties and the functionalities of components. Both the structure of the system and its components play essential roles in determining the symbiotic state of the system as a whole.

Within this context, Meyers (2012) outlines four key milestones that show how much we understand the system, namely, (a) to understand the structure of the system, such as gene regulatory and biochemical networks; (b) to understand the dynamics of the system using both quantitative and qualitative analyses, as well as the construction of a theory/model with a powerful prediction capability; (c) to understand the control methods of the system and (d) to understand the design methods of the system. The main objective of systems biology is the modeling and the discovery of emergent properties (Meyers, 2012).

Membrane systems have also been applied in the field of systems biology to model complex biological systems and analyze their dynamics. In their study, Besozzi et al. (2008) presented a class of membrane systems, where probabilities are associated with the rules governing the evolution of the system. They showed how this model can be used to describe an oscillating process, the Belousov-Zhabotinskii reaction. The synthetic 
Autoinducer-2 signaling system in genetically engineered Escherichia coli (E.coli) bacteria, which was modeled using membrane computing, has shown that a membrane computing model allows a user not only to monitor interactions at the level of an individual cell, but also to observe the emergent properties of entire cell populations (Esmaeili et al., 2009).

The attempts to model biological systems with membrane computing can be categorized into three, based on the context of biological systems. First, the modeling of biological systems involved in molecular reactions to changes in the environment or to changes caused by external stimulations of a certain pressure or a specific stimulus. The model of photosynthesis in chloroplast that deals with light reaction and photoinhibition and which was modeled by Nishida (2002) using membrane computing, belongs to this category. Second, the modeling of biological systems that prioritize the execution of interconnected processes in sequence, in which a process should first be completed before the next is executed. The research attempts in this category are the following: specifications of leukocyte selective recruitment in the immune system (Besozzi, 2003), descriptions of mechanosensitive channel behaviors in E. coli (Ardelean et al., 2005) and definitions of the sodium-potassium exchange pump in animal cells (Besozzi and Ciobanu, 2004). Third, the modeling of biological systems that involve emergent behaviors, in which interactions of processes within a compartment or interactions between compartments generate a global behavior. The comprehensive approaches on the aspects of quorum sensing in the bacteria Vibrio Fischeri (Romero-Campero and PerezJimenez, 2008a), the dynamics of HIV infection (Corne and Frisco, 2008), the robustness of the Epidermal Growth Factor Receptor (EGFR) in signaling cascade (Perez-Jimenez and Romero-Campero, 2005), gene expression control in Lac Operon (Romero-Campero and Perez-Jimenez, 2008b), the Ligand-Receptor network of the protein TGF- $\beta$, hormone-induced calcium oscillations in liver cells and the synthetic Autoinducer-2 signaling system in genetically engineered $E$. coli bacteria 3 (Esmaeili et al., 2009) are the biological systems in this category that have been modeled in membrane computing.

\subsection{Attempts to Integrate Membrane Computing with Other Paradigms}

Investigations into membrane computing paradigm demonstrate its perspectives, concepts and methods that model biological data, processes, as well as the system.
These characters and elements of membrane computing bring it closer to the modeling of biological systems that are compared with other natural computing paradigms. This is mainly because membrane computing could extract the data and the processes that are involved in biological systems, while simultaneously preserving the discrete and the stochastic behaviors of biological systems.

There are also other bio-inspired paradigms, for instance, the amorphous computing (Abelson et al., 2000), the self-healing system (George et al., 2003) and the General Model for Simulation of Dynamical Systems (MGS) (Giavitto and Michel, 2001), which provides a mechanism that represents data and processes in a specific way. Self-healing systems provide the mechanism for cell division, as well as chemical emission and diffusion. Amorphous computing offers a self-organizing system for population or tissue type systems. MGS presents the topological organization of a system.

A theoretical analysis of membrane computing (Muskulus and Brijder, 2006) has shown that the elements represented by the computer science paradigm could also be incorporated into membrane computing to facilitate various biological processes and behaviors. This is mainly because membrane computing embodies the essential cellular characteristics and the behaviors required by biological systems. The chemical emissions and the diffusions from one compartment to another, which are represented by self-healing systems, could be modeled using the communication rules of membrane computing. Bernardini and Gheorghe (2004) have shown that membrane computing could be used to perform cell divisions, as shown by self-healing systems. Membrane computing is also capable of modeling self-organization systems or of constructing emergent behaviors, as demonstrated by amorphous computing. The population or tissue type of membrane computing possesses these characteristics. Moreover, compartment rules, such as division, creation, dissolving, deletion, differentiation and binding rules (Bernardini and Gheorghe, 2004), which play a role in self-organizing systems biology, could also be implemented in population membrane computing. Membrane computing can also be organized topologically, although not parallel to cellular automata, which have fixed topological organizations. The objects and the rules are determined based on the membrane or the compartment where they are located. This mechanism could characterize the state of dynamic systems, such as in MGS. 
At the same time, there have been many research carried out to link membrane computing with other formalisms, for instance, the relationship of membrane computing with process algebra (Cardelli and Paun, 2006), neural networks (Ionescu et al., 2006), fuzzy logic (Casasnovas and Rossello, 2005), genetic algorithm (Escuela and Gutierrez-Naranjo, 2010) and Petri nets (Qi et al., 2004). These attempts could lead to a more comprehensive membrane computing to deal with the emerging challenges in systems biology. This means membrane computing provides flexibility in accommodating other concepts and approaches, which have been or are being researched in the fields of computational biology and systems biology.

\section{DISCUSSION}

Experiences in modeling aspects of systems biology with membrane computing demonstrate the various perspectives, concepts and methods being employed to represent data, processes, as well as the system and exhibit the usage of these representations to simulate various computations or to generate intended behavior. These approaches and concepts show additional advantages and possibilities compared with conventional methods. However, membrane computing, which is inspired by biology, is not yet used widely to model or simulate biological processes or systems, although it has advantages in mimicking those found in real natural systems. These include flexibility, adaptability, robustness and decentralized control. These are the fundamental aspects of membrane computing that imitate the behaviors found in biological systems.

These experiences show the possibilities of implementing membrane computing in biology again, where it originated from. The experiences of modeling biological systems, which were discussed above, show that biological systems could be modeled in a better way using membrane computing. This approach can counter some of the limitations in conventional methods, based on differential equations.

The experiences mentioned demonstrate that, so far, research in membrane computing in modeling biological systems have addressed the following biological elements: (a) process interactions and compartment interconnections; (b) discrete evolution of objects and processes; (c) non-deterministic and parallel execution of rules; (d) emergent behaviors based on the various process interactions in a compartment or communications among compartments; (e) representation of the structure of biological systems; (f) topological organizations based on structural arrangements of compartments; and ( $\mathrm{g}$ ) characterization of the stochastic behaviors of biological systems that involve interactions of processes among smaller number of objects or the microscopic elements of biological systems.

Membrane computing is a discrete computer science model, in which many things are done, such as clarifying the many mathematical problems that decorate the landscape of membrane computing and exploring the practical problems of applications at the same time. The framework is a rather general and versatilecompartmental computation, with separate "processors" communicating in various ways. They are synchronized, non-deterministic, parallel and dealing with multisets. They have an unlimited range of branches, analogies, cooperation with other areas and possible applications, as shown by the various attempts to incorporate membrane computing with other paradigms.

\section{CONCLUSION}

The categorization of biological elements that can be modeled in membrane computing shows that membrane computing is capable of abstracting biological structures and behaviors and representing them in a formal way without disregarding its biological characteristics. This also demonstrates that membrane computing could act as a better computational tool to address the investigations in systems biology.

However, there are still other elements in biological systems, such as how well membrane computing maintains biological behaviors in the interactions of a large number of objects and the capability of membrane computing to handle interruptions in biological events, that should be investigated by modeling them in membrane computing. The ODE approach is still regarded as a better approach in modeling biological systems that involve interactions of processes consisting of a large number of objects (Jong, 2002). Therefore, the capabilities of membrane computing in sustaining the behaviors of such biological systems should also be studied. Biological systems are also interrupted frequently by different biological events during the execution of processes (Degn et al., 1987). The capacity of membrane computing to handle such interruptions should also be investigated.

However, although membrane computing is proven theoretically to have computational completeness and computational efficiency, many of its aspects have not been tested in real problems. For instance, the elements in population membrane computing (Bernardini and 
Gheorghe, 2004), gemmation membrane computing (Besozzi et al., 2001) and membrane computing with symport-antiport (Paun et al., 2005; 2006) have not been used to model biological systems. This is so because, basically, there is no proper framework to model and to verify biological systems with membrane computing that could act as a guideline for researchers in computational biology or systems biology to use in exploring the advantages of membrane computing. Although research in modeling biological systems with membrane computing have proposed some guidelines in modeling and simulating membrane computing, they are limited to specific biological systems or processes.

Therefore, a general framework on modeling and verifying biological systems using membrane computing is required to provide guidelines for biologists and to encourage the use of membrane computing mechanisms to assist research in systems biology.

\section{REFERENCES}

Abelson, H., D. Allen, D. Coore, C. Hanson and G. Homsy et al., 2000. Amorphous computing. Commun. ACM, 43: 74-82. DOI: $10.1145 / 332833.332842$

Ardelean, I.I., D. Besozzi, M.H. Garzon, G. Mauri and S. Roy, 2005. P System models for mechanosensitive channels. Appli. Membrane Comput. DOI: 10.1007/3-540-29937-8_2

Bernardini, F. and M. Gheorghe, 2004. Population P systems. J. Universal Comput. Sci., 10: 509-539.

Besozzi, D. and G. Ciobanu, 2004. A P system description of the sodium-potassium pump. Membrane Comput., 3365: 210-223. DOI: 10.1007/978-3-540-31837-8_12

Besozzi, D., 2003. Computational and Modelling power of P systems. Ph.D. Thesis. Facolta di Scienze Matematiche Universita Degli Studi Di Milano Italy.

Besozzi, D., C. Zandron, G. Mauri and N. Sabadini, 2001. P systems with gemmation of mobile membranes. Theoretical Comput. Sci., 2202: 136153. DOI: 10.1007/3-540-45446-2_9

Besozzi, D., G. Mauri, D. Pescini and C. Zandron, 2008. Membrane systems in systems biology. Proceedings of the 9th International Workshop on Discrete Event Systems, May 28-30, IEEE Xplore Press, Goteborg, pp: 275-280. DOI: 10.1109/WODES.2008.4605959

Blanchard, P., R.L. Devaney and G.R. Hall, 2011. Differential Equations. 4th Edn., Brooks Cole, ISBN-10: 1133109039, pp: 864.
Cardelli, L. and G. Paun, 2006. An universality result for A (MEM) brane calculus based on mate/drip operations. Int. J. Foundations Comput. Sci., 17: 4968. DOI: $10.1142 / \mathrm{S} 0129054106003693$

Casasnovas, J. and F. Rossello, 2005. Fuzzy P systems and their applications in computational biology. University of the Balearic Islands.

Corne, D.W. and P. Frisco, 2008. Dynamics of HIV infection studied with cellular automata and conformon-P systems. Biosystems, 91: 531-544. DOI: 10.1016/j.biosystems.2007.01.007

Degn, H., A.V. Holden and L.F. Olsen, 1987. Chaos in Biological Systems. 1st Edn., Springer, New York, ISBN-10: 0306426854, pp: 323.

Escuela, G. and M.A. Gutierrez-Naranjo, 2010. An application of genetic algorithms to membrane computing. Proceedings of the 8th Brainstorming Week on Membrane Computing, (BWMC' 10), Sevilla, Spain, pp: 101-108.

Esmaeili, A., I. Yazdanbod and C. Jacob, 2009. A model of the quorum sensing system in genetically engineered E.coli using membrane computing. Proceedings of the International Genetically Engineered Machine (iGEM) Competition, Oct. 21-21.

George, S., D. Evans and S. Marchette, 2003. A biological programming model for self-healing. Proceedings of the 1st ACM Workshop on Survivable and Self-Regenerative Systems, Oct. 2730, ACM Press, Washington, DC, USA., pp: 72-80. DOI: $10.1145 / 1036921.1036929$

Giavitto, J.L. and O. Michel, 2001. MGS: A programming language for the transformation of topological collections. Technical Report, CNRS Universited'Evry Val d'Essonne.

Ionescu, M., G. Paun and T. Yokomori, 2006. Spiking neural P systems. Fundam. Inform., 71: 279-308.

Jong, H.D., 2002. Modeling and simulation of genetic regulatory systems: A literature review. J. Comput. Biol., 9: 67-103. DOI: 10.1089/10665270252833208

Meyers, R.A., 2012. Systems Biology. 1st Edn., John Wiley and Sons, ISBN-10: 3527326073, pp: 726.

Muskulus, M. and R. Brijder, 2006. Complexity of biocomputation: Symbolic dynamics in membrane systems. Int. J. Found. Comput. Sci., 17: 47-47. DOI: $10.1142 / \mathrm{S} 0129054106003747$

Nishida, T.Y., 2002. Simulations of photosynthesis by a K-subset transforming system with membrane. Fundam. Inform., 49: 249-259. 
Paun, G., G. Ciobanu and M.J. Perez-Jimenez, 2006. Application of Membrane Computing. Springer Verlag (Natural Computing Series), Berlin.

Paun, G., G. Rozenberg and A. Salomaa, 2010. The Oxford Handbook of Membrane Computing. 1st Edn., Oxford University Press, USA., ISBN-10: 0199556679, pp: 696.

Paun, G., M.J. Perez-Jimenez, J. Pazos and A. Rodriguez-Paton, 2005. Symport/antiport P systems with three objects are universal. Fundamenta Inform., 64: 353-367.

Perez-Jimenez, M.J. and F.J. Romero-Campero, 2005. Modelling EGFR signalling cascade using continuous membrane system. Proceedings of the 3rd Workshop on Computational Methods in System Biology, (CMSB’ 05).
Qi, Z., J. You and H. Mao, 2004. P systems and petri nets. Membrane Comput., 2933: 286-303. DOI: 10.1007/978-3-540-24619-0 21

Romero-Campero, F.J. and M.J. Perez-Jimenez, 2008a. A model of the quorum sensing system in vibrio fischeri using P systems. Artif. Life, 14: 95-109. DOI: 10.1162/artl.2008.14.1.95

Romero-Campero, F.J. and M.J. Perez-Jimenez, 2008b. Modelling gene expression control using $\mathrm{P}$ systems: The Lac operon, a case study. Biosystems, 91: 438457. DOI: 10.1016/j.biosystems.2007.02.011 